\title{
Colour Doppler and Biomarkers Utility for Renal Damage due to Congenital Hydronephrosis
}

\author{
Yan-Nan Guo, Xiu-Ying Chen and Li-Li Liu
}

\begin{abstract}
Objective: To determine colour doppler and serum biomarkers spectrum in children with congenital hydronephrosis.

Study Design: An observational study.

Place and Duration of Study: Department of Pediatric Nephrology, West China 2nd University Hospital of Sichuan University and Key Laboratory of Birth Defects and Related Disease of Women and Children (Sichuan University), China, from January to December 2017.

Methodology: A total of 95 children with hydronephrosis were selected as case group. According to the degree of hydronephrosis, the patients were divided into mild hydronephrosis group, moderate hydronephrosis group, and severe hydronephrosis group. Forty children with normal renal function were selected as normal comparison group. Peak systolic velocity $\left(\mathrm{V}_{\max }\right)$, end diastolic velocity $\left(\mathrm{V}_{\min }\right)$, resistance index $(\mathrm{RI})$, pulsatility index $(\mathrm{PI})$, and serum cystatin $\mathrm{C}(\mathrm{Cys})$, $\beta_{2}$-microglobulin $\left(\beta_{2}-M G\right)$, and $\alpha_{1}$-microglobulin $\left(\alpha_{1}-M G\right)$ of all subjects in both groups were recorded and compared.

Results: The $\mathrm{V}_{\max }, \mathrm{V}_{\max }$ of main renal artery (MRA) and interlobar renal artery (IRA) in case group were lower than those of normal group (all $p<0.001$ ). RI of MRA and IRA in case group were higher than those of normal control group (both $p<0.001)$. There were no significant differences in the PI of MRA and IRA between the two groups $(p=0.700$, and 0.250 respectively). The levels of serum CysC, $\beta_{2}-M G$ and $\alpha_{1}-M G$ in normal control group, mild hydronephrosis group, moderate hydronephrosis group, and severe hydronephrosis group were significantly different (all $p<0.001$ ), and the levels of serum CysC, $\beta_{2}-\mathrm{MG}, \alpha_{1}-\mathrm{MG}$ were also different in children with different degrees of hydronephrosis.

Conclusion: Combined detection of colour doppler and serum biomarkers CysC, $\beta_{2}-M G$ and $\alpha_{1}-M G$ in the diagnosis of renal damage in congenital hydronephrosis is feasible and reliable.
\end{abstract}

Key Words: Congenital hydronephrosis, Colour doppler, CysC, $\beta_{2}-M G, \alpha_{1}-M G$.

\section{INTRODUCTION}

Congenital hydronephrosis, as one of the most common urinary system abnormalities is often found in children with ureteropelvic junction obstruction. ${ }^{1-3}$ Finding effective, safe and minimally invasive indicators for congenital hydronephrosis evaluation has become a hotspot in current clinical research. Ultrasonography is an easy-to-use, non-invasive and non-radioactive means of examination that can detect morphological damage of hydronephrosis. However, using grayscale ultra-sonography to distinguish cases of mild hydronephrosis from normal cases showing fullness only, is relatively difficult.4-6 Studies show that the levels of serum biomarkers, such as cystatin $\mathrm{C}$ (CysC), $\beta_{2}$-micro-

Department of Pediatric Nephrology, West China $2^{\text {nd }}$ University, Hospital of Sichuan University and Key Laboratory of Birth

Defects and Related Disease of Women and Children (Sichuan University), Ministry of Education, Chengdu 610041, China

Correspondence: Li-Li Liu, Department of Pediatric

Nephrology, West China 2nd University, Hospital of Sichuan

University and Key Laboratory of Birth Defects and Related

Disease of Women and Children (Sichuan University),

Ministry of Education, Chengdu 610041, China

E-mail: ehdixecvcp8@163.com

Received: April 12, 2018; Accepted: August 15, 2018 globulin $\left(\beta_{2}-M G\right)$ and $\alpha_{1}$-microglobulin $\left(\alpha_{1}-M G\right)$ could be used to effectively evaluate renal function.7-8 Previous studies find that serum CysC, $\beta_{2}-\mathrm{MG}$ may be potential biomarkers of obstruction in hydronephrosis. ${ }^{9}$

The aim of this study was to evaluate the clinical value of colour doppler combined with serum CysC, $\beta_{2}-\mathrm{MG}$ and $\alpha_{1}-M G$ in the evaluation of congenital hydronephrosis, with a view to provide a reference for management of congenital hydronephrosis.

\section{METHODOLOGY}

This study was conducted at the Department of Pediatric Nephrology, West China Second University, Hospital of Sichuan University and Key Laboratory of Birth Defects and Related Disease of Women and Children (Sichuan University), Ministry of Education, Chengdu, China, from January to December 2017. A total of 95 children with hydronephrosis were selected as the case group. Inclusion criteria were patients with congenital hydronephrosis, no obvious abnormalities on grayscale ultrasound of the liver, gallbladder, pancreas, spleen, chest X-ray or heart and lung, who could tolerate the examination. Exclusion criteria were patients with immune dysfunction, severe liver and kidney damage, and urinary tract infections, recent history of trauma or surgery, or hydronephrosis caused by other causes such 
as urinary tract infections or tumors. Hydronephrosis was graded as mild on ultrasound when the renal collection system was $1.0-2.0 \mathrm{~cm}$ in dimension, and renal parenchyma and kidney shape was normal. Moderate hydronephrosis was ultrasound showing separation of the renal collection system by $2.1-3.5 \mathrm{~cm}$, and the renal parenchyma thinner, and kidney enlarged. Severe hydronephrosis was labelled when ultrasound showed separation range of the renal collection system $\geq 3.6 \mathrm{~cm}$, the kidney enlarged, and the renal parenchyma thinned by compression or completely shrunk. A total of 40 children with normal kidney function and having checkups in the hospital over the same period were selected as the normal control group. The study was approved by the Hospital Ethical and Research Committee, and family members of the subjects all signed the informed consents.

Colour doppler diagnostic system was used for examination. The abdominal convex array probe was used at a frequency of $3-5 \mathrm{MHz}$, sampling volume $3 \mathrm{~mm}$, wall filter $100 \mathrm{~Hz}$, and the angle between blood flow and acoustic beam $\leq 30^{\circ}$. First, the shape and various diameters of the kidneys were observed by twodimensional ultrasonography, and blood flow measurements were made after obtaining a standard coronal section. The sampling frame was placed at the main renal artery (MRA) of the renal hilum and the interlobar renal artery (IRA) of the renal parenchyma to obtain a

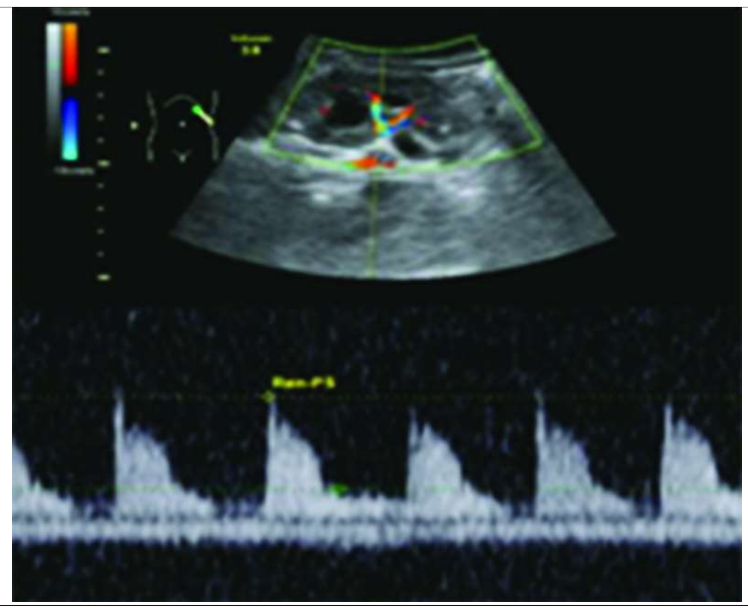

Figure 1: RI of intra renal arteries in children with hydronephrosis. satisfactory blood flow spectrum ( 3 to 5 consecutive waveforms similar to each other). The peak systolic velocity $\left(\mathrm{V}_{\max }\right)$, the end diastolic velocity $\left(\mathrm{V}_{\text {min }}\right)$, resistance index (RI), and pulsatility index (PI) were measured. The levels of CysC, $\beta_{2}-M G$ and $\alpha_{1}-M G$ in the serum of the subjects were measured by the double-antibody sandwich enzyme-linked immunosorbent assay (ELISA).

SPSS 21.0 statistical software was used for data analysis. Measurement data were expressed as mean \pm standard deviation. The independent samples t-test was used for comparison between two groups and oneway ANOVA was used for comparison among multiple groups. A difference with a $p$-value less than 0.05 was considered statistically significant.

\section{RESULTS}

The 95 children in the case group were divided into the mild hydronephrosis group 30 cases $(31.58 \%)$, the moderate hydronephrosis group 31 cases $(32.63 \%)$, and the severe hydronephrosis group 34 cases $(35.79 \%)$.

In case group, there were 60 males $(63.16 \%)$ and 35 females $(36.84 \%)$; aged from 4 months to 11 years, mean $5.26 \pm 0.97$ years; 46 cases $(48.42 \%)$ of ureteropelvic junction obstruction (UPJO), 40 cases $(42.11 \%$ ) of UVJO (ureteropelvic junction obstruction) and 9 cases $(9.47 \%)$ of ureteroceles; 50 cases $(52.63 \%)$ of unilateral hydronephrosis, 45 cases $(47.37 \%)$ of bilateral hydronephrosis; 58 cases $(61.05 \%)$ of hydronephrosis in the left kidney and 37 cases (38.95\%) of hydronephrosis in the right kidney. In normal control group, there were 25 males $(62.50 \%)$ and 15 females $(37.50 \%)$; aged 4 months to 10 years, mean $5.17 \pm 0.62$ years.

The $\mathrm{V}_{\text {max }}, \mathrm{V}_{\max }$ of main renal artery (MRA) and interlobar renal artery (IRA) in case group were lower than those of normal control group (all $p<0.001$ ). The RI of MRA and IRA in case group were higher than those of normal control group (both $p<0.001$ ). There were no significant difference in the PI of MRA and IRA between the two groups $(p=0.700$ and 0.250 , respectively, Table I and Figure 1).

The levels of serum CysC, $\beta_{2}-\mathrm{MG}$ and $\alpha_{1}-\mathrm{MG}$ in normal control group, mild hydronephrosis group, moderate hydronephrosis group, and severe hydronephrosis group were significantly different (all $\mathrm{P}<0.001$ ), and the

Table I: Comparison of kidney colour doppler parameters between case group and normal control group.

\begin{tabular}{|c|c|c|c|c|c|}
\hline \multirow[t]{2}{*}{ Parameter } & \multirow[t]{2}{*}{ Groups } & \multicolumn{2}{|c|}{ MRA } & \multicolumn{2}{|c|}{ IRA } \\
\hline & & Mean $\pm S D$ & $p$-value & Mean \pm SD & $\mathrm{p}$-value \\
\hline Vmax & $\begin{array}{l}\text { Normal control group }(n=40) \\
\text { Case group }(n=95)\end{array}$ & $\begin{array}{l}53.76 \pm 6.08 \\
40.12 \pm 3.26\end{array}$ & $<0.001$ & $\begin{array}{l}28.02 \pm 2.53 \\
14.08 \pm 1.30\end{array}$ & $<0.001$ \\
\hline Vmin & $\begin{array}{l}\text { Normal control group }(n=40) \\
\text { Case group }(n=95)\end{array}$ & $\begin{array}{l}24.18 \pm 2.94 \\
16.03 \pm 1.31 \\
\end{array}$ & $<0.001$ & $\begin{array}{l}13.92 \pm 1.47 \\
9.27 \pm 0.39 \\
\end{array}$ & $<0.001$ \\
\hline RI & $\begin{array}{l}\text { Normal control group }(n=40) \\
\text { Case group }(n=95)\end{array}$ & $\begin{array}{l}0.63 \pm 0.05 \\
0.74 \pm 0.06\end{array}$ & $<0.001$ & $\begin{array}{l}0.56 \pm 0.11 \\
0.71 \pm 0.05\end{array}$ & $<0.001$ \\
\hline $\mathrm{PI}$ & $\begin{array}{l}\text { Normal control group }(n=40) \\
\text { Case group }(n=95)\end{array}$ & $\begin{array}{l}1.26 \pm 0.19 \\
1.27 \pm 0.11\end{array}$ & 0.700 & $\begin{array}{l}1.21 \pm 0.14 \\
1.23 \pm 0.07\end{array}$ & 0.250 \\
\hline
\end{tabular}


Table II: Comparison of the levels of serum cys $C, \beta_{2}-M G$ and $\alpha_{1}-M G$ in each group.

\begin{tabular}{|c|c|c|c|c|c|c|c|}
\hline \multirow[t]{2}{*}{ Groups } & \multirow[t]{2}{*}{$\mathrm{n}$} & \multicolumn{2}{|c|}{ CysC (mg/L) } & \multicolumn{2}{|c|}{$\beta_{2}-\mathrm{MG}(\mathrm{mg} / \mathrm{L})$} & \multicolumn{2}{|c|}{$\alpha_{1}-M G(m g / L)$} \\
\hline & & Mean \pm SD & $\mathrm{p}$-value & Mean $\pm S D$ & $\mathrm{p}$-value & Mean \pm SD & p-value \\
\hline Normal control group & 40 & $0.79 \pm 0.05$ & $<0.001$ & $1.82 \pm 0.32$ & $<0.001$ & $19.93 \pm 2.04$ & $<0.001$ \\
\hline Mild hydronephrosis group & 30 & $0.92 \pm 0.10$ & & $2.16 \pm 0.71$ & & $23.07 \pm 5.20$ & \\
\hline Moderate hydronephrosis group & 31 & $1.01 \pm 0.26$ & & $3.04 \pm 0.63$ & & $25.02 \pm 4.28$ & \\
\hline Severe hydronephrosis group & 34 & $1.09 \pm 0.41$ & & $5.54 \pm 0.96$ & & $26.73 \pm 5.22$ & \\
\hline
\end{tabular}

levels of serum CysC, $\beta_{2}$-MG and $\alpha_{1}$-MG were also different in children with different degrees of hydronephrosis (Table II).

\section{DISCUSSION}

Congenital obstruction is the most common cause of congenital hydronephrosis. Congenital obstruction can lead to poor urine excretion, increase the expansion pressure in the pelvis, compress the renal parenchyma, and cause changes in multiple vascular factors such as angiotensin II in the kidney, then it will cause renal vasoconstriction, promote a further increase of renal vascular resistance and decrease renal function.10,11 Without timely treatment, congenital hydronephrosis may cause renal failure. So it is extremely important to determine the degree of obstruction and renal function in clinical practice.12,13 The conventional B-scan ultrasonography can easily detect the extent of water accumulation, but it cannot evaluate renal function; Intravenous pyelography (IVP) and radioisotope renography can be used to understand renal function; but in cases of severe hydronephrosis, IVP cannot visualise the calyceal system because of poor excretion of contrast.14,15 The results of this study showed that there were significant differences in the $\mathrm{V}_{\max }, \mathrm{V}_{\min }$ and $\mathrm{RI}$ under colour doppler between normal control group and case group. The $\mathrm{V}_{\max }$ and $\mathrm{V}_{\min }$ in case group were both lower than those of normal control group, while there were no significant difference in the PI of MRA and IRA between the two groups. The RI of the MRI and IRA in case group were both higher than those of normal control group, suggesting that RI was of practical significance as an indicator of vascular resistance. This conclusion is in accordance with the view proposed by Kawai et al. ${ }^{16}$

According to the related research results, the expression of $\mathrm{CysC}$ was closely related to the glomerular filtration rate, and it could be used as an objective indicator to evaluate the glomerular filtration function. 17 Parvex et al. concluded that CysC was a promising marker of renal function, at birth, in neonates prenatally diagnosed with congenital kidney anomalies. ${ }^{18}$ The results of this study were shown that the levels of serum CysC in normal control group, mild hydronephrosis group, moderate hydronephrosis group, and severe hydronephrosis group had statistically significant differences; and the levels of serum CysC in children with different degrees of hydronephrosis were higher than those in normal control group. This illustrates that the $\mathrm{CysC}$ is an important biomarkers to assess congenital hydronephrosis.

Elevated serum $\beta_{2}-M G$ levels suggested a decrease in glomerular filtration rate, while elevated urinary $\beta_{2}-\mathrm{MG}$ levels reflected damaged renal tubular reabsorption function. ${ }^{19}$ Bartoli et al. substantiated that the role of $\beta_{2}-M G$ as marker of tubulointerstitial damage in human obstructive nephropathy. 20 The results of this study suggested that children's damaged glomerular function was caused by the inability of normal glomeruli to compensate for $\beta_{2}-M G$ excretion; the results also indicated that the serum $\beta_{2}$-MG levels were different in children with varying degrees of hydronephrosis and the degrees of glomerular function damage would also vary. This study found that $\beta_{2}-\mathrm{MG}$ was a promising marker of congenital hydronephrosis.

The protein $\alpha_{1}-\mathrm{MG}$ is a glycoprotein with small molecular weight, it is mainly synthesised in liver and lymphoid tissues. ${ }^{21}$ It is widely present in various body fluids of the human body and can freely pass through the glomerular filtration membrane. However, most of such proteins are reabsorbed and decomposed by proximal convoluted tubules. Under normal circumstances, $\alpha_{1}-M G$ is rarely seen in urine. Its content in urine can only increase when the renal tubular is injured. So, it is currently widely believed that $\alpha_{1}-\mathrm{MG}$ is an indicator to reflect glomerular and renal tubular functions. The results of this study demonstrated that the serum $\alpha_{1}$-MG levels could well indicate whether the renal tubule was damaged; and the severer the damage was, the higher the $\alpha_{1}$-MG level would be.

It can be seen that the detection on the levels of serum CysC, $\beta_{2}-M G$ and $\alpha_{1}-M G$ can well distinguish cases of mild hydronephrosis from normal cases, and can make up for the deficiency of kidney ultrasonography in this aspect. Therefore, colour doppler combined with serum CysC, $\beta_{2}-M G$ and $\alpha_{1}-M G$ has important clinical significance on the evaluation of congenital hydronephrosis. At the same time, this study also has many limitations and shortcomings; for instance, the number of the studies is less, and future still has many aspects to perfect.

\section{CONCLUSION}

Combined detection of colour doppler and serum biomarkers CysC, $\beta_{2}-\mathrm{MG}$ and $\alpha_{1}-\mathrm{MG}$ in the diagnosis of 
renal damage in congenital hydronephrosis is feasible and reliable.

\section{REFERENCES}

1. Pogorelic Z, Brkovic T, Budimir D, Todoric J, Košuljandic D, Jeroncic A, et al. Endoscopic placement of double-J ureteric stents in children as a treatment for primary hydronephrosis. Can J Urol 2017; 24:8853-8.

2. Zee RS, Herbst KW, Kim C, Mckenna PH, Bentley T, Cooper CS, et al. Urinary tract infections in children with prenatal hydronephrosis: a risk assessment from the society for fetal urology hydronephrosis registry. J Pediatr Urol 2016; 12:262-3.

3. Petrovski M, Simeonov R, Todorovikj L, Chadikovski V, Memeti S, Petrovska B, et al. Congenital hydronephrosis: disease or condition? Makedon Akad Nauk Umet Odd Med Nauki 2014; 35:123-9.

4. Choi YH, Cheon JE, Kim WS, Kim IO. Ultrasonography of hydronephrosis in the newborn: A practical review. Ultrasonography 2016; 35:198-211.

5. Bratslavsky G, Kogan BA. Febrile urinary tract infections in infants: renal ultrasound remains necessary. J Urol 2005; 173:568-70

6. Patel MD, Young SW, Scott KJ, Dahiya N. Ultrasound-guided renal transplant biopsy: practical and pragmatic considerations. Abdom Radiol (NY) 2018; 2:1-7.

7. Hossein-Emad M, Arash D, Mohammad K. Correlation of cystatin $C$ and creatinine-based estimates of renal function in children with hydronephrosis. J Renal Inj Prev 2016; 5:25-8.

8. Rao X, Wan M, Qu C, Jiang C. Role of cystatin C in renal damage and the optimum cut-off point of renal damage among patients with type 2 diabetes mellitus. Exp Ther Med 2014; 8: $887-92$

9. Madsen MG, Nørregaard R, Palmfeldt J, Olsen LH, Frøkiær J, Jørgensen TM. Urinary NGAL, cystatin $C, \beta_{2}$-microglobulin, and osteopontin significance in hydronephrotic children. Pediatric Nephrol 2012; 27: 2099-106.

10. Pugacevska D, Groma V, Petersons A, Melderis I, Gilis A. Morphological changes of ureteropelvic junction (UPJ) in case of congenital hydronephrosis - review of literature. Acta Chirurgica Latviensis 2013; 13:72-5.

11. Smith BG, Metwalli AR, Leach J, Cheng EY, Kropp BP. Congenital midureteral stricture in children diagnosed with antenatal hydronephrosis. Urology 2004; 64:1014-9.

12. Gupta M, Gupta M, Abhishek. Oral conditions in renal disorders and treatment considerations - A review for pediatric dentist. Saudi Dent J 2015; 27:113-9.

13. Xu ZM, Li MJ, Tao C. Serum and urinary thioredoxin concentrations are associated with severity of children hydronephrosis. Clinica Chimica Acta 2017; 466:127-32.

14. Li X, Liu X, Li J, Song E, Sun N, Liu W, et al. Semaphorin-3a and netrin-1 predict the development of kidney injury in children with congenital hydronephrosis. Scand J Clin Lab Invest 2017; 78:1-7.

15. Heon CW, Duck CJ, Ho K K, Jin SY, Kyungseop L. Comparison and efficacy of low-dose and standard-dose tamsulosin and alfuzosin in medical expulsive therapy for lower ureteral calculi: prospective, randomized, comparative study. Korean J Urol 2012; 53:349-54.

16. Kawai T, Kamide K, Onishi M, Yamamoto-Hanasaki H, Baba Y, Hongyo $\mathrm{K}$, et al. Usefulness of the resistive index in renal doppler ultrasonography as an indicator of vascular damage in patients with risks of atherosclerosis. Nephrol Dial Transplant 2011; 26:3256-62.

17. Uemura O, Nagai T, Ishikura K, Ito S, Hataya H, Gotoh $\mathrm{Y}$, et al. Cystatin C-based equation for estimating glomerular filtration rate in japanese children and adolescents. Clin Exp Nephrol 2014; 18:718-25

18. Parvex P, Combescure C, Rodriguez M, Girardin E. Is cystatin C a promising marker of renal function, at birth, in neonates prenatally diagnosed with congenital kidney anomalies? Nephrol Dial Transplant 2012; 27:3477-82.

19. Foster MC, Hwang SJ, Larson MG, Parikh NI, Meigs JB, Vasan RS, et al. Cross-classification of microalbuminuria and reduced glomerular filtration rate: associations between cardiovascular disease risk factors and clinical outcomes. Arch Intern Med 2007; 167:1386-92.

20. Bartoli F, Penza R, Aceto G, Niglio F, DAddato O, Pastore V, et al. Urinary epidermal growth factor, monocyte chemotactic protein-1, and $\beta_{2}$-microglobulin in children with ureteropelvic junction obstruction. J Pediatr Surg 2011; 46:530-6.

21. Hadjidekov G, Hadjidekova S, Tonchev Z, Bakalova R, Aoki I. Assessing renal function in children with hydronephrosisadditional feature of MR urography. Radiol Oncol 2011; 45: 248-58. 